\title{
VLBI CORES IN A SAMPLE OF RADIO GALAXIES
}

\author{
G. Comoretto, 1 L. Feretti,2,3 G. Giovannini 2,3 \\ 1 - Osservatorlo Astrofisico di Arcetri, L.go E. Fermi 5, \\ Firenze, Italy \\ 2 - Istituto di Radloastronomia, v. Irnerio 46, Bologna \\ 3 - Dipartimento di Astronomia, v. Zamboni 33, Bologna, Italy
}

We present the first results of a statistical study of the milliarcsec structure in a complete sample of radio galaxles. We have selected from the B2 and 3CR samples of galaxies the sources which present, at the VLA or WSRT angular resolution, an unresolved core with a flux density at $5 \mathrm{GHz} \mathrm{S}_{c} \geq 100 \mathrm{mJy}$. The total sample consists of 30 radio galaxies, 17 from the $B 2$ and 13 from the $3 C R$ catalog. This complete sample covers a range of total radio power at $408 \mathrm{MHz}$ $\log \mathrm{P}=23.5-26.5 \mathrm{~W} / \mathrm{Hz}$ (low-intermediate luminosity). The radio structure of these sources on the arcsec-arcmin scale is well known, thanks to good dynamic range VLA and or WSRT maps; a large varlety of structures is present in the sample, from classical doubles to head-tall sources; flat, inverted and steep spectrum cores are also present.

The 12 radio galaxies with the strongest cores have been already observed by other authors with the VLBI, therefore we observed at $5 \mathrm{GHz}$ the remaining 18 galaxies with the EVN array + Haystack antenna, using the Mark III recording system in mode B. As a first step, each source was observed with 1 to 8 scans of 13 minutes in order to have indications on the size and flux of the nuclear sources. Unambiguous mapping is not possible because of the limlted $u-v$ coverage, but crude Indlcations about the core structure can be derived. Thanks to the transatlantic baselines, we were able to detect structure down to a resolution of 2 mas in $E-W$ direction.

The results are summarized in table 1 . We detected 15 out of the 18 sources. In 9 sources all the arcsec flux was detected on the VLBI scale, in 4 sources more than $70 \%$; only in 2 sources less than 65\% of the arcsec flux was detected. This result is different from that of Wehrle et al. (1984, Ap. J., 284, 519), who observed on the baseline Goldstone-Madrid a complete sample of radio galaxies, selected according to the total flux at $408 \mathrm{MHz}$. They found that for most sources the flux in the VLBI component is only a fraction of the flux detected in the arcsec core. This Implles that besides the compact feature some resolved structure, whlch can be missed in observations with only transatlantic baselines, is generally present in these sources.

M. J. Reid and J. M. Moran (eds.), The Impact of VLBI on Astrophysics and Geophysics, 75-76.

(c) 1988 by the IAU. 
In our sample, of the 15 sources detected, 8 are unresolved and 7 show indication of an extended structure. The limited $u-v$ coverage available allow us to derive only simple models. All sources show a structure of the type core-jet, with the parsec jet well alligned with the Kpc jet. In one source $(0755+37)$ a jet-counterjet is strongly suggested both in the map and in the model.

The accurate mapping with better $u-v$ coverage of all the detected sources is planned as a second step of this work.

\section{TABLE 1}

Summary of observations

\begin{tabular}{|c|c|c|c|c|c|c|}
\hline \multicolumn{2}{|c|}{ Source name } & \multirow{2}{*}{\begin{tabular}{c}
\multicolumn{2}{c}{ Beam } \\
mas (deg) \\
$6 \times 2(0)$
\end{tabular}} & \multirow{2}{*}{\begin{tabular}{|c}
$\begin{array}{c}\text { Scans } \\
\text { observed }\end{array}$ \\
6
\end{tabular}} & \multirow{2}{*}{$\begin{array}{c}\begin{array}{c}\text { Number of } \\
\text { stations }\end{array} \\
6\end{array}$} & \multirow{2}{*}{\begin{tabular}{|l|} 
Structure \\
one sided
\end{tabular}} & \multirow{2}{*}{$\begin{array}{c}\begin{array}{c}S_{\text {mas }} / S_{a s} \\
\%\end{array} \\
100\end{array}$} \\
\hline $0055+30$ & NGC315 & & & & & \\
\hline $0104+32$ & 3 C31 & $6 \times 2 \quad(0)$ & 6 & 6 & point & 100 \\
\hline $0220+43$ & 3C66B & $5 \times 2 \quad(60)$ & 2 & 6 & point & 100 \\
\hline $0222+36$ & & $6 \times 2 \quad(20)$ & 2 & 6 & extended? & 65 \\
\hline $0258+35$ & NGC1167 & $5 \times 2$ & 2 & 6 & - & $<20$ \\
\hline $0331+39$ & 4C39.12 & $6 \times 2 \quad(30)$ & 1 & 6 & point & 85 \\
\hline $0755+37$ & NGC2484 & $4 \times 2 \quad(0)$ & 8 & 6 & two sided & 100 \\
\hline $0836+29$ & & $4 \times 2 \quad(-15)$ & 6 & 6 & one sided & 100 \\
\hline $1037+30$ & & $4 \times 2 \quad(-15)$ & 6 & 6 & - & $<7$ \\
\hline $1142+20$ & $3 \mathrm{C} 264$ & $8 \times 2 \quad(0)$ & 4 & 6 & one sided? & 90 \\
\hline $1144+35$ & & $6 \times 2 \quad(15)$ & 2 & 6 & one sided & variable \\
\hline $1322+36$ & NGC5141 & $4 \times 2 \quad(30)$ & 2 & 6 & point & 50 \\
\hline $1441+52$ & 3C303 & $6 \times 2 \quad(-45)$ & 3 & 6 & point & 100 \\
\hline $1511+26$ & 3C315 & $14 \times 4(30)$ & 1 & 3 & 一 & $<5$ \\
\hline $1626+39$ & 3C338 & $7 \times 2 \quad(-15)$ & 5 & 6 & extended & 100 \\
\hline $1641+17$ & $3 \mathrm{C346}$ & $12 \times 6(-45)$ & 2 & 5 & point & 70 \\
\hline $1833+32$ & 3C382 & $8 \times 3 \quad(0)$ & 1 & 6 & extended? & 100 \\
\hline $2249+39$ & $3 \mathrm{C} 452$ & $3 \times 2 \quad(0)$ & 3 & 6 & one sided & 100 \\
\hline
\end{tabular}

